\title{
The Principal Component Analysis of Tribological Tests of Surface Layers Modified with IF-WS ${ }_{2}$ Nanoparticles
}

\section{PIETRASZEK Jacek ${ }^{1, a ~ *}$, KORZEKWA Joanna ${ }^{2, b}$, GOROSHKO Andrii ${ }^{3, c}$}

\author{
${ }^{1}$ Institute of Applied Informatics, Cracow University of Technology, \\ Poland, 31-864 Krakow, Al.Jana Pawła II 37, \\ ${ }^{2}$ Faculty of Computer and Materials Science, University of Silesia, \\ Poland, 41-200 Sosnowiec, ul.Sniezna 2 \\ ${ }^{3}$ Khmelnitsky National University \\ Ukraine, 29016 Khmelnitsky, Institutskaja 11
}

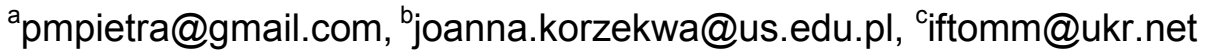

Keywords: large datasets, design of experiments, analysis of variance, principal component analysis, geometric structure parameters, surface layer, nano-particles

\begin{abstract}
The investigation described in this paper resulted in some complicated statistical analysis. The first level was an experimental design with technological parameters as factorials input and geometrical surface layer properties as quantitative outputs. The second level was an analysis generally leading to an optimization inverse problem: what parameters result in desired surface layer properties. The principal component analysis was made to identify possibility of a dimensionality reduction and simplify the optimization. Obtained results showed that the experimental dataset is practically two-dimensional but PCA projection involves all factors into the skewed hyper-plane. This paper contains a description of the problem, obtained results, analysis and conclusions.
\end{abstract}

\section{Introduction}

The aim of investigation is the analyze of geometric structure parameters of tribological layers $\mathrm{Al}_{2} \mathrm{O}_{3} /$ IF-WS $\mathrm{W}_{2}$ obtained by two methods of deposition of the inorganic fullerene-like nano-powder of the tungsten disulfide IF-WS . The layers of $\mathrm{Al}_{2} \mathrm{O}_{3} / \mathrm{IF}-\mathrm{WS}_{2}$ were obtained in the two-stage production process. In a first step, the $\mathrm{Al}_{2} \mathrm{O}_{3}$ layer was prepared in the process of anodic oxidation in a three-component electrolyte. The second stage involved the introduction of a layer of IF-WS nanopowder in the nano-pores and on the surface using dipping method. Ethanol and ethylene glycol were liquid dispersion for a nanopowder. The resulting layers were coupled with a thermoplastic polyetherketone of fillers: graphite, carbon fiber and PTFE-called PEEK / BG, under the conditions of dry friction on the tester T17, working in reciprocating motion. Measurements of the structure geometric parameters SGP of $\mathrm{Al}_{2} \mathrm{O}_{3} / \mathrm{IF}-\mathrm{WS}_{2}$ were performed using a contact profilograph Taylor Hobson Talysurf 3D and the results were subjected to principal component analysis.

Aluminum alloys are valued construction material because of its good corrosion resistance, high impact strength at low temperatures, and the preferred ratio of strength to specific weight. Due to the very high affinity for the adhesive tacks, however, are not suitable for direct use them as the cooperating parts of machinery and equipment. One method of eliminating the adverse impact of adhesive tacks on the surfaces of co-operating aluminum components of a machinery is is the application of the method basing on the anodic oxidation of alloys surface. Layers, obtained by such a method, are used as a protective coatings for machine elements such as actuators and pistons engines. In recent years, many research studies have been devoted to modify the surface of the aluminum layer in order to reduce the coefficient of friction between the frictional elements. Anodic oxide coatings owe their good tribological properties to appropriate surface structure, which is created by micro and nanopores of layer. Properties of the surface layers are often described using 
the parameters of a geometric structure that reflects their topography and morphology. Using the advance statistical methods, this paper presents an analysis of the results obtained during studies of the geometric structure of a surface. This study provides new information on the anodic oxide layer doped with tungsten disulfide, made using a two-step technological process.

\section{Materials and methods}

The anodic oxidation of aluminum. In the first stage of the process, the aluminum oxide layer was formed on a base of alloy EN AW 5251 using chemical electrolysis. Before carrying out the electrolysis, the samples were digested in $5 \% \mathrm{KOH}$ solution for $45 \mathrm{~min}$, then $10 \% \mathrm{HNO}_{3}$ solution for $10 \mathrm{~min}$. The anodic oxidation was performed on aluminum plates having a surface $10^{-3} \mathrm{~m}^{2}$ in solution of acids: sulfuric, oxalic acid and phthalic, for $1 \mathrm{~h}$ at a current density of $3 \mathrm{~A} / \mathrm{dm}^{2}$, at a constant temperature of $303 \pm 1 \mathrm{~K}$.

Modification of $\mathbf{A l}_{2} \mathbf{O}_{3}$ layers. IF-WS2 nanopowder (Nanomaterials Ltd) was introduced into the nanoporous $\mathrm{Al}_{2} \mathrm{O}_{3}$ layer using the dipping method with an ultrasonic bath. Nanopowder IF-WS2 was dispersed in the two solutions: 35 vol. \% solution of ethanol $\left(\mathrm{C}_{2} \mathrm{H}_{6} \mathrm{O}\right)(\operatorname{method} 1)$ and 35 vol. \% solution of ethylene glycol $\left(\mathrm{C}_{2} \mathrm{H}_{6} \mathrm{O}_{2}\right)$ (method 2). To analyze the reproducibility of tribological test results, three samples were prepared from each method and an unmodified sample for comparison.

Measurements of parameters of the geometrical structure. The output for the analysis of SGP were the parameters: $R a, R q, R p, R v, R t, R s k, R k u$ and $R z$. These parameters were measured at three locations of the sample (Fig. 1): in the $X$ direction - the sample surface before friction; in the direction $\mathrm{Y}$ - the sample surface after the friction at the point of adhesion tacks; in the direction $\mathrm{Z}-$ the sample surface after friction.

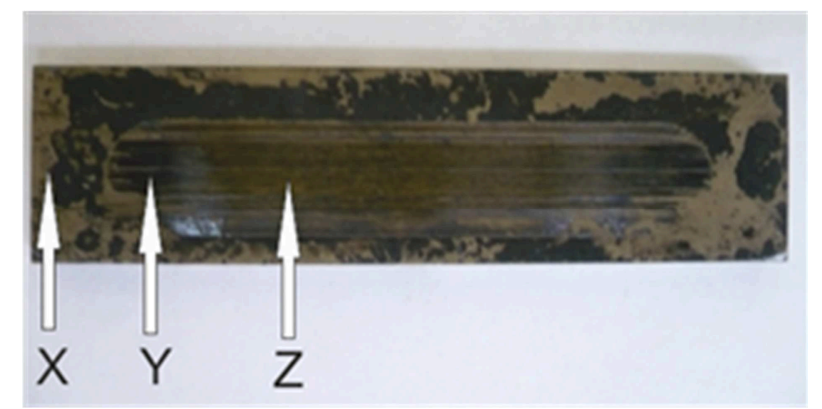

Fig. 1. The picture showing the location of the SGP measurement:

$\mathrm{X}$ - sample surface before friction,

$\mathrm{Y}$ - the sample surface after friction in place of adhesive tacks,

$\mathrm{Z}$ - the sample surface after friction.

SGP measurements of oxide layers were made by the Taylor Hobson Talysurf 3D pin profilometer with the accuracy of $2 \%$. The results of the parameters were developed by using the TalyMap Universal 3D software. The stereometric analysis was performed on an area of $2 \mathrm{~mm} \times 2 \mathrm{~mm}$. The investigated surface of the slide film was obtained by combination of the surface layers with plastic PEEK/BG (polyetheretherketone with PTFE, graphite and carbon fibers of ERTA). Tribological test was conducted under conditions of dry friction in reciprocating motion on the T17 tester (produced by ITeE-PIB Radom). The tribological test was conducted for $15 \mathrm{~km}$. The fresh cross section structure of the $\mathrm{Al}_{2} \mathrm{O}_{3} / \mathrm{IF}-\mathrm{WS}_{2}$ layer were analyzed by a HITACHI S-4700 scanning electron microscope (SEM) with a NORAN Vantage digital energy dispersive X-ray microanalysis (EDS) system. The samples were coated by thin gold film, using a sputtering apparatus in order to improve the image contrast and prevent blurring by charge accumulation. The surface of the $\mathrm{Al}_{2} \mathrm{O}_{3} / \mathrm{IF}_{-}-\mathrm{WS}_{2}$ layer were analyzed by Bruker Dimension Edge atomic force microscope (Labsoft).

Statistical analysis. The results of measurements of surface texture was analyzed by the method of the principal components analysis (PCA) $[1,2]$, which is one of the dimensionality reduction methods in the group of the linear discriminant analysis methods (LDA). 


\section{Results}

The raw data contains 630 records of 8 quantitative geometric properties: Ra, Rq, Rp, Rv, Rt, Rsk, Rku, Rz classified by two factors: 'group' (unmodified, ethanol, ethylene glycol) and 'position' ( $\mathrm{x}, \mathrm{y}, \mathrm{z})$. Numbers of measurements in particular classification bins are presented in Table 1. Descriptive statistics of the whole dataset is presented in Table 2.

Table 1. Number of measurements in particular classification bins

\begin{tabular}{|c|c|c|c|c|}
\hline & unmodified & ethanol & ethylene glycol & Total \\
\hline $\mathbf{x}$ & 30 & 90 & 90 & 210 \\
\hline $\mathbf{y}$ & 30 & 90 & 90 & 210 \\
\hline $\mathbf{z}$ & 30 & 90 & 90 & 210 \\
\hline Total & 90 & 270 & 270 & 630 \\
\hline
\end{tabular}

Table 2. Descriptive statistics of raw dataset

\begin{tabular}{|l|r|r|r|r|r|r|r|r|}
\hline Property & \multicolumn{1}{|c|}{ Mean } & \multicolumn{1}{c|}{ Median } & Minimum & Maximum & \multicolumn{1}{c|}{ Q1 } & \multicolumn{1}{c|}{ Q3 } & \multicolumn{1}{c|}{ S } & \multicolumn{1}{c|}{ SE } \\
\hline $\mathrm{Ra}$ & 1.30 & 0.77 & 0.31 & 12.35 & 0.49 & 1.11 & 1.54 & 0.06 \\
\hline $\mathrm{Rq}$ & 1.69 & 1.00 & 0.41 & 14.87 & 0.67 & 1.50 & 1.94 & 0.08 \\
\hline $\mathrm{Rp}$ & 4.20 & 2.58 & 0.90 & 30.32 & 1.43 & 4.27 & 4.29 & 0.17 \\
\hline $\mathrm{Rv}$ & 3.61 & 2.27 & 1.01 & 24.02 & 1.69 & 3.12 & 3.83 & 0.15 \\
\hline $\mathrm{Rt}$ & 16.92 & 9.23 & 3.57 & 162.67 & 7.01 & 21.06 & 17.66 & 0.70 \\
\hline $\mathrm{Rsk}$ & 1.92 & 1.32 & -2.37 & 26.09 & 0.53 & 2.42 & 2.76 & 0.11 \\
\hline $\mathrm{Rku}$ & 21.99 & 10.44 & 3.27 & 401.32 & 6.70 & 20.49 & 37.44 & 1.49 \\
\hline $\mathrm{Rz}$ & 7.80 & 4.83 & 2.20 & 54.34 & 3.29 & 7.20 & 7.95 & 0.32 \\
\hline
\end{tabular}

\section{Analysis}

The following principal component analysis (PCA) shows that the method of imposition of nanolubricant on the surface of pure $\mathrm{Al}_{2} \mathrm{O}_{3}$ affects the parameters of the geometric structure. PCA data set consisting of $\mathrm{N}$ observations, each of which includes $\mathrm{K}$ quantitative variables can be interpreted as a cloud of $\mathrm{N}$ points in k-dimensional hyperspace. For the analysis presented in the article, $\mathrm{N}=30$ or $\mathrm{N}=90$ observations means 30 or 90 measurements and $\mathrm{K}=8$ means the eight analyzed parameters describing the structure $(R a, R q, R p, R v, R t, R s k, R k u$, and $R z)$. Finally, it means 630 data points in 8-dimensional hyperspace.

The structure of the analyzed parameters (variables) were subjected to linear transformations creating new coordinates called PCA factors, chosen to be uncorrelated, while the greatest variability of data cloud focuses on the least number of factors. On the basis of the scree plot (Fig. 2), it was determined that the first two PCA factors, designated as PCA1 and PCA2, are responsible for $96 \%$ of the data cloud variability. 


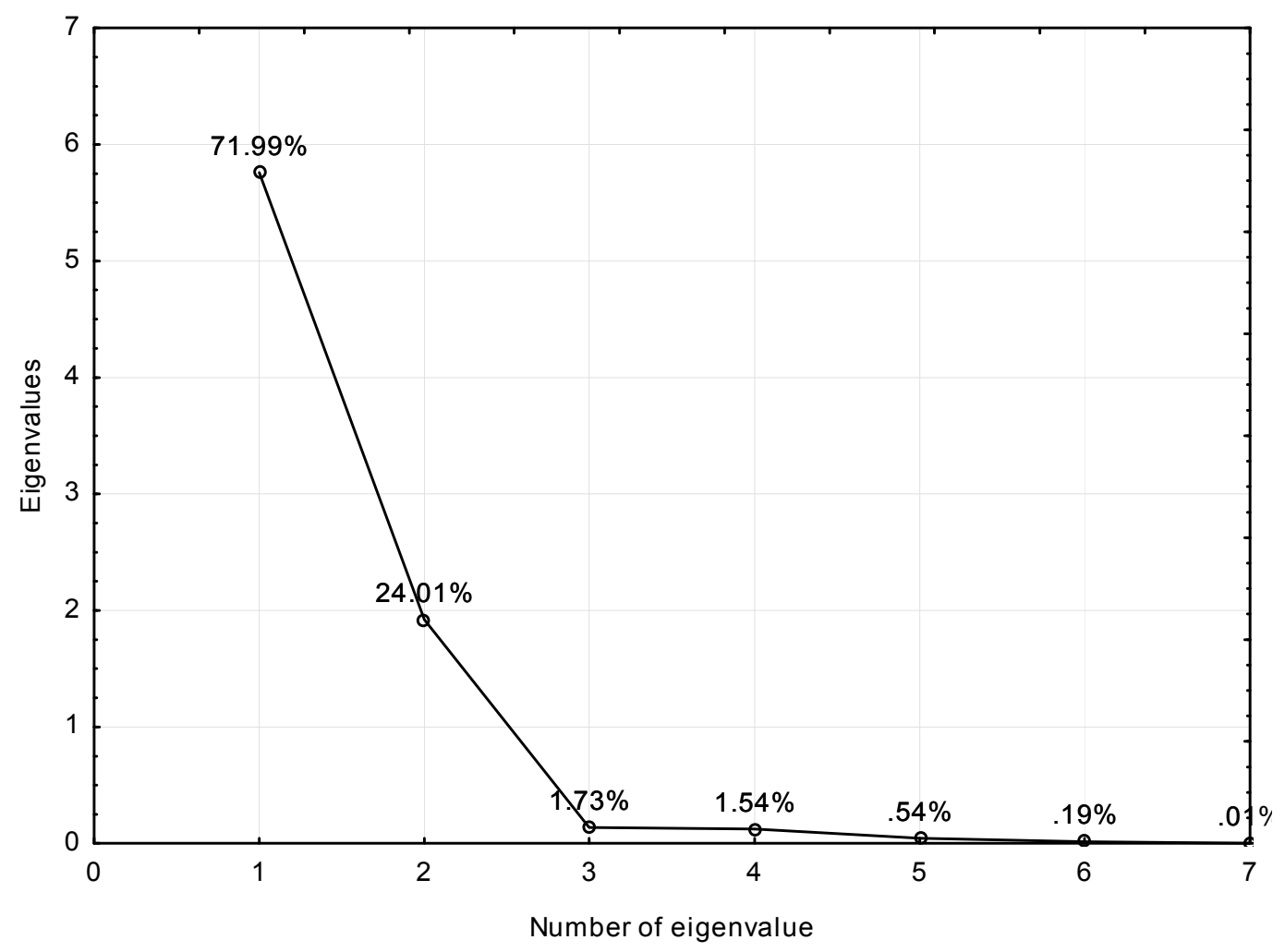

Fig. 2. Scree plot obtained from PCA analysis.

At the same time, the specific location of the PCA factors in relation to the original coordinates (structure parameters) presented on the load plot (Fig. 3) allows one to make some important observations and interpretations. It turns out that the dominant factor PCA1 almost overlaps with the parameter $R a$, and PCA2 factor - with parameter $R q$.

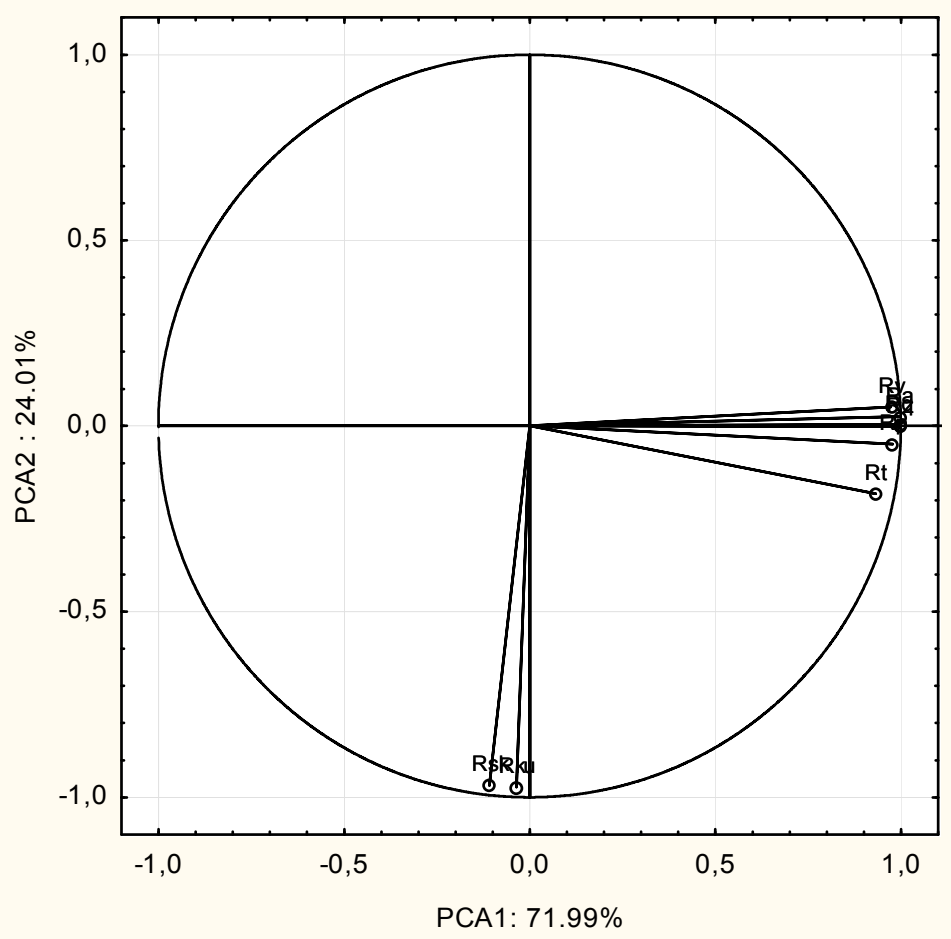

Fig. 3. Load plot obtained from PCA analysis. 
The linear relationships between surrogate factors PCA1/PCA2 and real geometric properties of the surface layer are quantitatively presented in Table 3 and visually in Fig. 3. As can be seen, the first six properties tends to form PCA1 and remaining two forms PCA2.

Table 3. Coefficients of linear relationships between PCA1/PCA2 and geometry properties of the surface layer

\begin{tabular}{|c|c|c|}
\hline \multirow{2}{*}{ Property } & \multicolumn{2}{|c|}{ Coefficient } \\
\cline { 2 - 3 } & PCA1 & PCA2 \\
\hline $\mathrm{Ra}$ & 0.994806 & 0.025068 \\
\hline $\mathrm{Rq}$ & 0.997282 & 0.003779 \\
\hline $\mathrm{Rp}$ & 0.977880 & -0.048384 \\
\hline $\mathrm{Rv}$ & 0.972395 & 0.051386 \\
\hline $\mathrm{Rt}$ & 0.931040 & -0.182491 \\
\hline $\mathrm{Rz}$ & 0.996712 & -0.001355 \\
\hline $\mathrm{Rsk}$ & -0.108666 & -0.967436 \\
\hline $\mathrm{Rku}$ & -0.035751 & -0.972521 \\
\hline
\end{tabular}
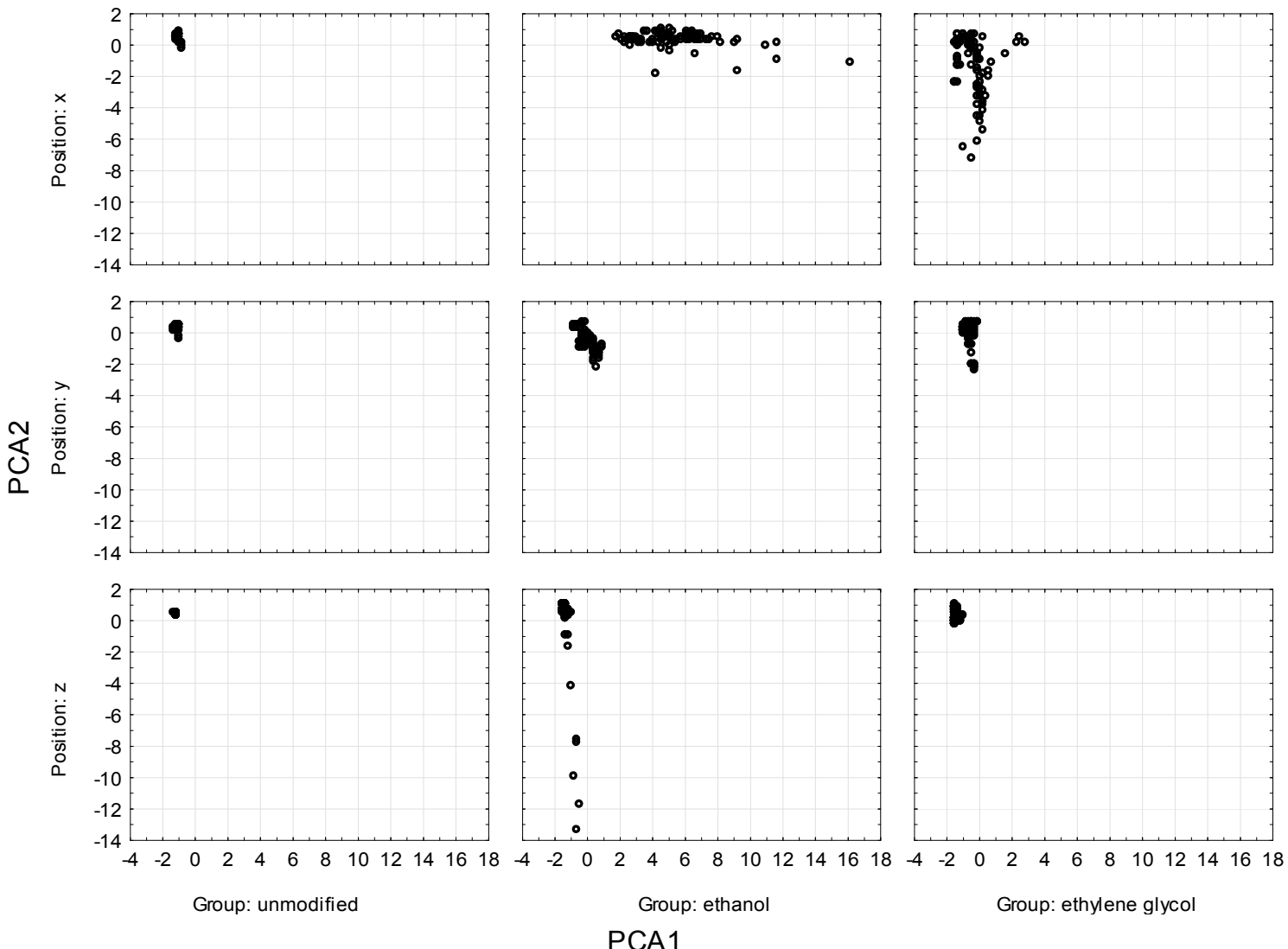

Fig. 4. Principal components analysis for the structure parameters the projection on the sub-space of factors PCA1 vs. PCA2.

According to relationship presented in Table 3, a variability along PCA1 axis means proportional variability of parameters $\mathrm{Ra}, \mathrm{Rq}, \mathrm{Rp}, \mathrm{Rv}, \mathrm{Rt}$ and $\mathrm{Rz}$. Analogically, a variability along PCA2 axis means proportional variability of parameters Rsk and Rku. There is a slight interference between both groups but this effect is negligible. 
Based on the analysis of the scatterplots (Fig. 4) for the projective sub-space PCA1 vs. PCA2, it can be concluded that there is practically no variability for unmodified material. There is almost the same variability along the PCA2 axis for both modified materials (ethanol and ethylene glycol) after the friction.

The significance difference between modified materials appears for positon $\mathrm{x}$ what means a zone before the friction. Nanopowders dispersed in the ethanol gave the large variability along the axis PCA1 (Ra, Rq, Rp, Rv, Rt and Rz) and rather small along the axis PCA2 (Rsk and Rku). Nanopowders dispersed in the ethylene glycol gave a reversed situation: the large variability along the axis PCA2 (Rsk and Rku) and small along the axis PCA1 (Ra, Rq, Rp, Rv, Rt and Rz).

\section{Conclusions}

The simplicity of the PCA interpretation is possible due to the particular configuration of the geometric properties on the PCA load plot. Six properties are almost parallel to the PCA1 factors, remaining two - to the PCA2 factor. Shapes of the clusterization, observed in zone ' $x$ ' for both modified surface layers, showed that the same nanopowders form surfaces with completely different characteristics depending on kind of solvent: ethanol vs. ethylene glycol.

Matrix categorized plot has been exceptionally useful tool for interpretation of PCA results classified by two factors with three labels each. This methodology can be useful in analogical issues of multidimensional data analysis in the field of the surface layer technology [3, 4], a quantitative evaluation of superalloys [5, 6], an image analysis for detection of production errors [7], a selfoscillating detection system [8], fuzzy systems [9, 10] and 2D or 3D image analysis [11]. The analysis of fracture surfaces [12] may be also a domain with possibilities of this method application.

However, there is still some doubt about PCA objectiveness [2]. This procedure may be evaluated by a covariance matrix or a correlation matrix and different results are obtained leading to different inference. Some articles related to such problems of objectiveness and stability were published [13], especially about data driven methods $[14,15]$.

\section{Summary}

The test of dry friction was made between specific plastic and surface layer modified by nanopowders dispersed in different solvents: ethanol and ethylene glycol. Control test was made on the unmodified surface layer. The 8 geometrical properties of the surface layer were scanned by a profilograph in three different zones: a surface outside of friction slide, a surface with tacles at reciprocating move and a surface at sliding move. Total number of 630 record of 8 measurements were collected and analyzed by the principal component analysis. The analysis revealed that a whole dataset may be described by two surrogate factors PCA1 and PCA2 explaining 96\% of total variability. Six geometric properties were highly correlated with PCA1 and remaining two with PCA2. Distinctive patterns were observed in the projection onto the plane PCA1 vs. PCA2. A deeper explanation of the meanings of these patterns is the issue addressed to materials science and it is beyond the scope of this article.

\section{Acknowledgements}

The authors would like to thank the support of Mr. S. Kaptacz on performing the SGP measurements.

\section{References}

[1] A.J. Izenman, Modern Multivariate Statistical Techniques. Regression, Classification and Manifold Learning, Springer Science+Business Media, LLC, New York 2008.

[2] I.T. Jolliffe, Principal Component Analysis, Springer, New York 2010.

[3] N. Radek, K. Bartkowiak, Laser Treatment of Cu-Mo Electro-Spark Deposited Coatings, Physcs. Proc. 12 (2011) 499-505. 
[4] M. Scendo, N. Radek, J. Trela, Influence of Laser Treatment on the Corrosive Resistance of WC-Cu Coating Produced by Electrospark Deposition, Int. J. Electrochem. Sc. 8/7 (2013) 9264-9277.

[5] A. Szczotok, On gamma-gamma' eutectic quantitative evaluation in the as-cast CMSX-4 nickel-based superalloy, Solid State Phenomen. 197 (2013) 203-208.

[6] K. Trzewiczek, A. Szczotok, A. Gadek-Moszczak, Evaluation of the State for The Material of the Live Steam Superheater Pipe Coils of V Degree, Adv. Mater. Res.-Switz. 874 (2014) 35-42.

[7] J. Pietraszek, A. Gadek-Moszczak, T. Torunski, Modeling of Errors Counting System for PCB Soldered in the Wave Soldering Technology, Adv. Mater. Res.-Switz. 874 (2014) 139-143.

[8] J. Kwasniewki, I. Dominik, K. Lalik, Application of self-oscillating system for stress measurement in metal, J. Vibroeng. 14/1 (2012) 61-66.

[9] I. Dominik, Implementation of the Type-2 Fuzzy Controller in PLC, Sol. St. Phen. 164 (2010) 95-98.

[10]J. Pietraszek, E. Skrzypczak-Pietraszek, The Optimization of the Technological Process with the Fuzzy Regression, Adv. Mater. Res.-Switz. 874 (2014) 151-155.

[11]H. Altendorf, F. Latourte, D. Jeulin, M. Faessel, L. Saintoyant, 3d Reconstruction of a Multiscale Microstructure by Anisotropic Tessellation Models, Image Anal. Stereol. 33/2 (2014) 121-130.

[12]L. Wojnar, M. Kumosa, Advanced Quantitative Analysis of Fracture Surfaces, Mat. Sci. Eng. A 128/1 (1990) 45-53.

[13]F. Camerlenghi, V. Capasso, E. Villa, Numerical Experiments for the Estimation of Mean Densities of Random Sets, Image Anal. Stereol. 33/2 (2014) 83-94.

[14]M. Baaske, F. Ballani, K.G. van den Boogaart, A Quasi-Likelihood Approach to Parameter Estimation for Simulatable Statistical Models, Image Anal. Stereol. 33/2 (2014) 107-119.

[15]M.H. Mozaffari, S.H. Zahiri, Unsupervised Data and Histogram Clustering Using Inclined Planes System Optimization Algorithm, Image Anal. Stereol. 33/1 (2014) 65-74. 